Ciência Florestal, Santa Maria, v. 21, n. 1, p. 23-30, jan.-mar., 2011

ISSN 0103-9954

\title{
BESOUROS CERAMBYCIDAE ASSOCIADOS A Eucalyptus spp. NO MUNICÍPIO DE PINHEIRO MACHADO, RS
}

\author{
CERAMBYCIDAE BEETLES ASSOCIATED TO Eucalyptus spp. IN THE MUNICIPALITY OF \\ PINHEIRO MACHADO, RS
}

\author{
Oderlei Bernardi ${ }^{1}$ Mauro Silveira Garcia ${ }^{2}$ Eduardo José Ely e Silva ${ }^{3}$ \\ Luiza Cristiane Fialho Zazycki ${ }^{4}$ Daniel Bernardi ${ }^{5}$ Dinorvan Miorelli ${ }^{6}$ Élder Finkenauer ${ }^{7}$
}

\section{RESUMO}

Este trabalho teve como objetivo coletar, identificar e caracterizar faunisticamente a entomofauna de Cerambycidae ocorrente em um plantio de Eucalyptus spp., fazenda São Manoel, município de Pinheiro Machado, RS. No período de fevereiro de 2006 a outubro de 2007, a cada 15 dias, foram realizadas coletas de insetos com três armadilhas etanólicas. Após procedimentos de triagem, os Cerambycidae foram identificados comparando-os com coleções entomológicas e com uso de literatura especializada. Foram coletados 692 insetos, distribuídos em 29 gêneros e quarenta espécies. As espécies mais abundantes foram Acanthoderes jaspidea, Chlorida costata, Compsocerus barbicornis, Eburodacrys sp., Eurysthea hirta, Neoclytus curvatus e Nyssodrysina lignaria, os quais representam $81,17 \%$ do total de indivíduos. Os cerambicídeos foram coletados em praticamente todo o período de amostragem, com pico populacional verificado no mês de dezembro. Várias espécies demonstram potencial de danificar Eucalyptus spp., sendo Neoclytus curvatus aquela que merece maior atenção, pois representou praticamente metade dos insetos coletados.

Palavras-chaves: Coleoptera; essência florestal; armadilha etanólica; análise faunística.

\begin{abstract}
The objective of this study was to collect, identify and carry out a faunistic characterization of Cerambycidae occurring in a forest of Eucalyptus spp., on São Manoel farm, in the municipality of Pinheiro Machado, RS. In the period from February 2006 to October 2007, collections of insects were performed every 15 days, with three ethanol traps. After selection procedures, the Cerambycidae were identified based on entomological collections and specialized literature. There were collected 692 insects, distributed among 29 genera and 40 species. The most abundant species were Acanthoderes jaspidea, Chlorida costata, Compsocerus barbicornis, Eburodacrys sp., Eurysthea hirta, Neoclytus curvatus and Nyssodrysina lignaria, which

1. Engenheiro Agrônomo, Doutorando em Entomologia, Escola Superior de Agricultura "Luiz de Queiroz", ESALQ/ USP, Av. Pádua Dias 11, Caixa Postal 9, CEP 13418-900, Piracicaba (SP). oderleibernardi@yahoo.com.br;

2. Engenheiro Agrônomo, Dr., Professor do Departamento de Fitossanidade, Faculdade de Agronomia Eliseu Maciel, Universidade Federal de Pelotas, Caixa Postal 354, CEP 96010-900, Pelotas (RS). garciasmauro@yahoo.com.br;

3. Engenheiro Agrônomo, Dr., Taxonomista, Departamento de Fitossanidade, Faculdade de Agronomia Eliseu Maciel, Universidade Federal de Pelotas, Caixa Postal 354, CEP 96010-900, Pelotas (RS). eduelysilva@uol.com.br;

4. Engenheira Agrônoma, Mestranda em Entomologia, Departamento de Fitossanidade, Faculdade de Agronomia Eliseu Maciel, Universidade Federal de Pelotas, Caixa Postal 354, CEP 96010-900, Pelotas (RS). luizazazycki@gmail.com;

5. Engenheiro Agrônomo, Mestrando em Entomologia, Departamento de Fitossanidade, Faculdade de Agronomia Eliseu Maciel, Universidade Federal de Pelotas, Caixa Postal 354, CEP 96010-900, Pelotas (RS). dbernardi2004@yahoo.com.br;

6. Engenheiro Agrônomo, Departamento de Fitossanidade, Faculdade de Agronomia Eliseu Maciel, Universidade Federal de Pelotas, Caixa Postal 354, CEP 96010-900, Pelotas (RS).dm.aratibars@yahoo.com.br;

7. Técnico Agrícola, Votorantim Celulose \& Papel S/A, Unidade Extremo Sul, BR 116, Km 532, CEP 96160-000, Capão do Leão (RS). elder.finkenauer@vcp.com.br

Recebido para publicação em 19/05/2009 e aceito em 12/01/2010.
\end{abstract}


represented $81.17 \%$ of the total individuals. Cerambycidae were collected during practically the entire sampling period, but the largest number at a single collection occurred in the month of December. Several species demonstrate potential for damaging Eucalyptus spp., of which Neoclytus curvatus deserves the most attention, because it represented almost half of the insects collected.

Keywords: Coleoptera; forest plant; ethanol trap; faunistic analysis.

\section{INTRODUÇÃO}

No Rio Grande do Sul, toda a metade sul do Estado está sendo alvo de intensos investimentos de empresas do setor florestal, a grande maioria voltados ao cultivo de eucalipto. Atualmente, o Estado, ocupa a sexta posição em área cultivada no País com espécies do gênero Eucalyptus, com 222.245 ha de um total de 3,7 milhões de hectares (ABRAF, 2008).

Com o aumento recente das áreas de cultivo de eucalipto na Região Sul do Brasil, ocorre um aumento considerável na disponibilidade de alimento para as espécies de insetos que utilizam o eucalipto para seu desenvolvimento, desse modo, paralelamente ao incremento da área de cultivo, acredita-se que aumentem também os problemas entomológicos. De acordo com Anjos et al. (1986), as espécies de eucalipto têm como principais pragas as formigas cortadeiras, os lepidópteros desfolhadores e os coleópteros, os quais constituem sérios problemas para a eucaliptocultura nacional.

Dentre os coleópteros que ocorrem associados às espécies florestais nativas e exóticas, causando algum tipo de dano, destacam-se pelo grande número de espécies e pelo alto grau de polifagia, os Cerambycidae (MARTINS, 2005).

Especificamente no Rio Grande do Sul, estudos envolvendo levantamento de espécies de Cerambycidae são escassos. Foram realizados trabalhos por Garcia e Corseuil (1998) que analisaram a flutuação populacional dos insetos dessa família na cultura do pessegueiro. Neto e Link (1997) estudaram a fauna de Cerambycidae associada às Lauráceas na região Central do Rio Grande do Sul. Biezanko e Bosq (1956) realizaram um estudo de cerambicídeos de Pelotas e arredores dando grande contribuição para o reconhecimento da fauna de Cerambycidae da região. No entanto, existem poucas informações referentes às espécies de Cerambycidae ocorrentes em florestamentos de eucalipto da Região Sul do Brasil.

Nesse sentido, o objetivo deste trabalho foi coletar, identificar e caracterizar faunisticamente a entomofauna de Cerambycidae ocorrente em um florestamento de Eucalyptus spp. localizado no município de Pinheiro Machado, RS.

\section{MATERIAL E MÉTODOS}

As coletas foram realizadas no período de fevereiro de 2006 a outubro de 2007, em área florestada com Eucalyptus spp., pertencente à empresa Votorantim Celulose \& Papel S/A, fazenda São Manoel, localizada às margens da rodovia BR293 a 7 km da cidade de Pinheiro Machado, estado

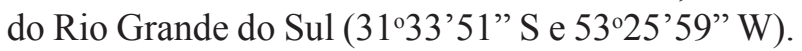

Foram utilizadas três armadilhas etanólicas, modelo ESALQ-84, que originalmente são de plástico, no entanto, estas foram confeccionadas com chapas de aço galvanizadas de $2 \mathrm{~mm}$ de espessura, pois são mais resistentes aos efeitos do ambiente. As armadilhas foram instaladas a $1,5 \mathrm{~m}$ de altura, tendo como referência a base do cone de captura conforme Flechtmann et al. (1995) e iscadas com álcool comercial (96\%), com a função de atrair os insetos. Na porção inferior da armadilha, foi fixado um frasco de vidro contendo álcool $70 \%$, com a função de coletar os insetos atraídos e interceptados (FLECHTMANN et al., 2000).

A distância entre cada armadilha foi de aproximadamente $200 \mathrm{~m}$ sendo colocadas a $50 \mathrm{~m}$ da borda em direção ao interior do florestamento. $\mathrm{O}$ período de captura foi de aproximadamente 15 dias, quando todos os insetos eram retirados do frasco coletor e o álcool renovado.

A identificação foi realizada pelo taxonomista Dr. Eduardo José Ely e Silva mediante comparação com exemplares do Museu Entomológico "Ceslau Biezanko" do Departamento de Fitossanidade (DFs) da Faculdade de Agronomia Eliseu Maciel (FAEM), Universidade Federal de Pelotas (UFPel) e por meio de bibliografia especializada (MARTINS, 1999; MARTINS, 2005). A análise faunística foi realizada pelo programa AnaFau (MORAES et al., 2003).

\section{RESULTADOS E DISCUSSÃO}

De fevereiro de 2006 a outubro de 2007 foram realizadas 41 coletas, sendo obtidos 692 
indivíduos, distribuídos em 40 espécies. Das espécies coletadas 36 foram identificadas por gênero e/ou espécie e quatro identificadas somente por família, isso perfaz 90,0 e $10,0 \%$ do total de indivíduos coletados respectivamente (Tabela 1). Esses valores são maiores que os encontrados por Freitas et al. (2002), que usando armadilhas luminosas, coletaram apenas 22 Cerambycidae em plantio de Eucalyptus grandis em Santa Bárbara, MG. Isso sugere que a abundância de Cerambycidae varia com a região, bem como é significantemente afetada pelo método de amostragem utilizado. As armadilhas luminosas são menos eficientes na coleta de espécies de Cerambycidae, fato que explica o baixo número de Cerambicydae coletados por Freitas et al. (2002). Essa constatação também foi feita por Carvalho (1984), em trabalho de coleta com armadilhas etanólicas e luminosas, o qual coletou grande número de cerambicídeos em povoamentos de Eucalyptus urophylla e Eucalyptus saligna no município de Salto, SP, sendo a maior quantidade observada em armadilhas etanólicas.

As espécies de Cerambycidae apresentaram picos populacionais nos meses de temperatura mais elevada, sendo o mês de dezembro aquele em que se coletou maior número de insetos, com 218 indivíduos (Figura 1). Resultados semelhantes foram verificados por Woldan (2007) em levantamento de Cerambycidae em mata nativa no município de União da Vitória, PR, onde verificou que o pico populacional dos insetos dessa família também ocorreu no mês de dezembro, quando foram registradas temperaturas mais elevadas.

TABELA 1: Número e porcentagem de Cerambycidae coletados com três armadilhas etanólicas em um plantio de Eucalyptus spp. no município de Pinheiro Machado, RS. Fevereiro de 2006 a outubro de 2007.

TABLE 1: Number and percentage of Cerambycidae collected with three ethanol traps on a plantation of Eucalyptus spp. in the municipality of Pinheiro Machado, RS. February 2006 to October 2007.

\begin{tabular}{ccccc}
\hline \multirow{2}{*}{ Grupo } & \multicolumn{2}{c}{ Espécies } & \multicolumn{2}{c}{ Indivíduos } \\
\cline { 2 - 5 } & Total & $\%$ & Total & $\%$ \\
\hline Identificados por gênero e/ou espécie & 36 & 90,0 & 632 & 91,33 \\
Identificados somente por família & 4 & 10,0 & 60 & 8,67 \\
\hline Total & 40 & 100,00 & 692 & 100,00 \\
\hline
\end{tabular}

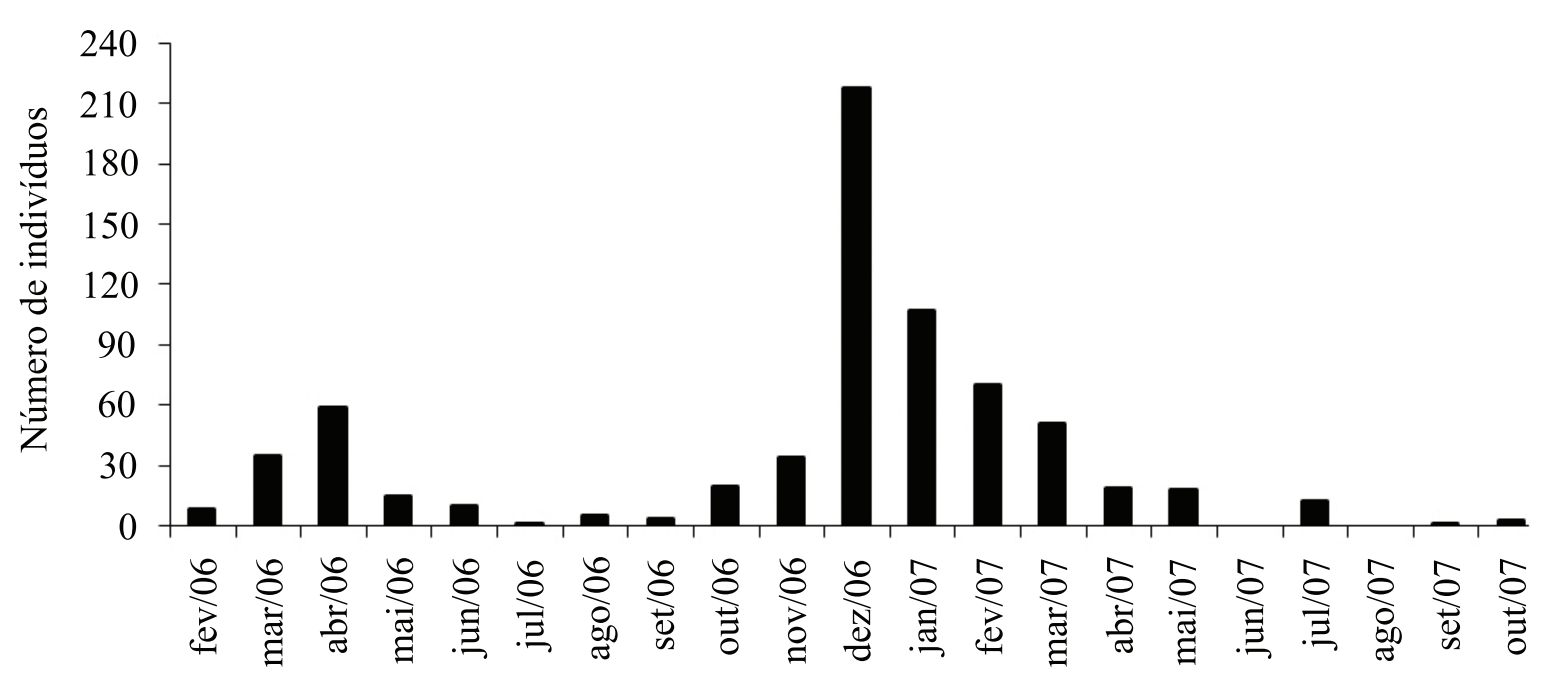

Período

FIGURA 1: Flutuação populacional de Cerambycidae coletados com três armadilhas etanólicas em um plantio de Eucalyptus spp. no município de Pinheiro Machado, RS. Fevereiro de 2006 a outubro de 2007.

FIGURE 1: Population fluctuation of Cerambycidae collected with three ethanol traps on a plantation of Eucalyptus spp. in the municipality of Pinheiro Machado, RS. February 2006 to October 2007. 
Bernardi, O. et al.

É importante salientar que as espécies Nyssodrysina lignaria e Neoclytus curvatus foram as responsáveis pela maioria dos picos populacionais observados no decorrer do estudo (Figura 2). Em trabalho com armadilhas Malaise, Marinoni e Ganho (2003) estudaram a sazonalidade de Nyssodrysina lignaria no estado do Paraná onde demonstraram que esta apresenta maior abundância de indivíduos nos meses de primavera e verão, semelhantemente ao observado no presente estudo. No entanto, Costa e Link (1988), em coletas com armadilhas etanólicas em eucalipto em Santa Maria, RS, verificaram que os picos populacionais de Nyssodrysina lignaria em eucalipto ocorreram em outubro e novembro, diferentemente dos verificados para o município de Pinheiro Machado (Figura 2).

De acordo com Marinoni e Ganho (2003), a flutuação populacional de Nyssodrysina lignaria é influenciada pela temperatura e umidade do ar. $\mathrm{Na}$ região de Pinheiro Machado, o pico populacional de Nyssodrysina lignaria em dezembro é produto de uma condição climática favorável com temperatura elevada, maior período de luz diária e ao provável período reprodutivo da espécie. Também pode estar associado à disponibilidade de frutos de árvores nativas, como espécies de ingá (Inga spp.), que são fontes alimentares de larvas dessa espécie (MARINONI et al., 2002).

Foram consideradas espécies predominantes por obterem os maiores índices de classificação faunística, como super frequente, super abundante, muito frequente, muito abundante, dominante e constante as espécies Acanthoderes jaspidea, Chlorida costata, Compsocerus barbicornis, Eburodacrys sp., Eurysthea hirta, Neoclytus curvatus e Nyssodrysina lignaria, perfazendo $79,58 \%$ do total de insetos identificados por gênero e/ou espécie (Tabela 2).

Dentre as espécies predominantes Neoclytus curvatus foi a espécie que apresentou maior número de indivíduos coletados (Figura 2). Essa espécie foi encontrada danificando plantas de Eucalyptus grandis e Eucalyptus globulus na Província de Lavalleja no Uruguai como relatado por Monné et al. (2002). Como os florestamentos apresentam expansão recente no Rio Grande do Sul, a espécie Neoclytus curvatus necessita ser monitorada com o intuito de acompanhar o comportamento de sua população com o decorrer dos anos de cultivo.

Foi verificada a presença de outras duas espécies do gênero, Neoclytus pussilus e Neoclytus ypsilon, as quais ocorrem em menor quantidade comparativamente a Neoclytus curvatus, sendo faunisticamente consideradas espécies dominantes, comuns, frequentes e acessórias. Neoclytus pussilus é citada no Brasil por Zanuncio et al. (1993), como potente broqueadora de troncos de Eucalyptus pellita, Eucalyptus tereticornis e Eucalyptus urophylla, demonstrando bom estabelecimento em áreas de cultivo de espécies do gênero Eucalyptus, fato também comprovado por Berti Filho (1997) que verificou aumento na população do inseto em Eucalyptus pellita no estado de Minas Gerais.

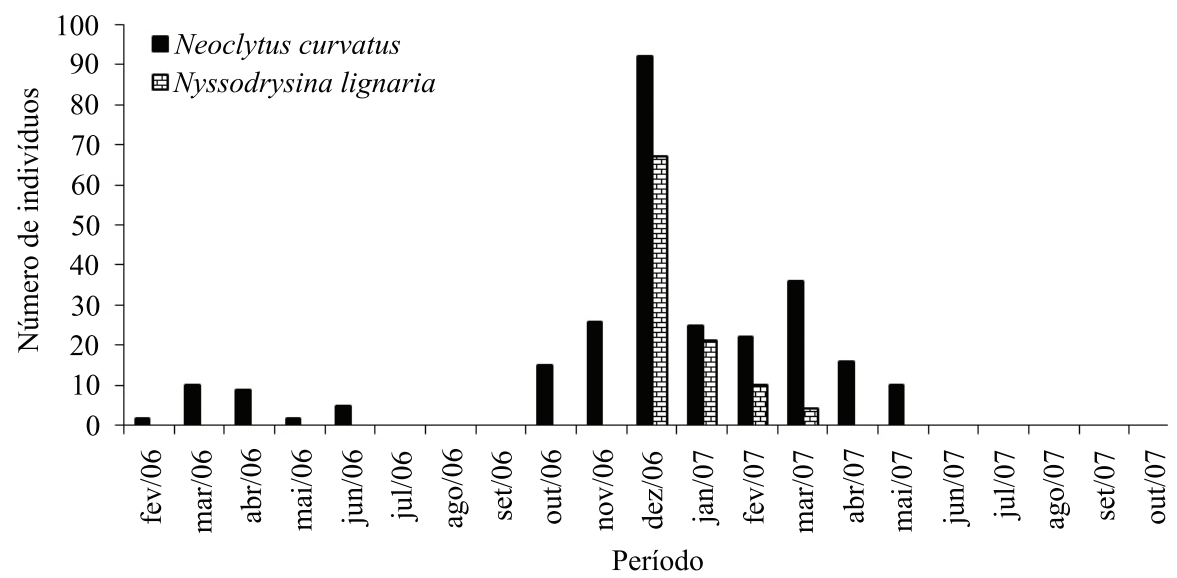

FIGURA 2: Flutuação populacional de Neoclytus curvatus e Nyssodrysina lignaria coletados com três armadilhas etanólicas em florestamento de Eucalyptus spp. no município de Pinheiro Machado, RS. Fevereiro de 2006 a outubro de 2007.

FIGURE 2: Population fluctuation of Neoclytus curvatus and Nyssodrysina lignaria collected with three ethanol traps on a plantation of Eucalyptus spp. in the municipality of Pinheiro Machado, RS. February 2006 to October 2007.

Ci. Fl., v. 21, n. 1, jan.-mar., 2011 
TABELA 2: Análise faunística de Cerambycidae coletados com três armadilhas etanólicas em plantio de Eucalyptus spp. no município de Pinheiro Machado, RS. Fevereiro de 2006 a outubro de 2007.

TABLE 2: Faunistic analysis of Cerambycidae collected with three ethanol traps on a plantation of Eucalyptus spp. in the municipality of Pinheiro Machado, RS. February 2006 to October 2007.

\begin{tabular}{|c|c|c|c|c|c|c|}
\hline Espécies & Indivíduos & $\%$ & $\mathrm{D}^{1}$ & A & $\mathrm{F}$ & $\mathrm{C}$ \\
\hline Acanthoderes jaspidea (Germar, 1824) & 23 & 3,64 & $\mathrm{~d}$ & ma & $\mathrm{mf}$ & $\mathrm{w}$ \\
\hline Acanthoderes nigricans Lameere, 1885 & 3 & 0,47 & nd & $\mathrm{d}$ & $\mathrm{pf}$ & $\mathrm{z}$ \\
\hline Achryson surinamum (Linnaeus, 1767) & 3 & 0,47 & nd & d & $\mathrm{pf}$ & $\mathrm{y}$ \\
\hline Centrocerum elegans (Chevrolat, 1861) & 2 & 0,32 & nd & $\mathrm{r}$ & $\mathrm{pf}$ & $\mathrm{z}$ \\
\hline Chlorida costata Audinet-Seville, 1834 & 23 & 3,64 & d & ma & $\mathrm{mf}$ & $\mathrm{w}$ \\
\hline Chlorida festiva Linneus, 1758 & 7 & 1,11 & d & $\mathrm{c}$ & $\mathrm{f}$ & $\mathrm{y}$ \\
\hline Chydarteres striatus striatus (Fabricius, 1787) & 8 & 1,27 & d & $\mathrm{c}$ & $\mathrm{f}$ & $\mathrm{w}$ \\
\hline Compsoceridius gounelle (Bruch, 1908) & 1 & 0,16 & nd & $\mathrm{r}$ & $\mathrm{pf}$ & $\mathrm{z}$ \\
\hline Compsocerus barbicornis Audinet-Serville, 1834 & 45 & 7,12 & d & ma & $\mathrm{mf}$ & $\mathrm{w}$ \\
\hline Compsocerus violaceus (White,1853) & 2 & 0,32 & nd & $\mathrm{r}$ & $\mathrm{pf}$ & $\mathrm{z}$ \\
\hline Dorcacerus barbatus (Olivier, 1790) & 1 & 0,16 & nd & $\mathrm{r}$ & $\mathrm{pf}$ & $\mathrm{z}$ \\
\hline Eburodacrys sp. & 20 & 3,16 & d & ma & $\mathrm{mf}$ & $\mathrm{w}$ \\
\hline Erosida lineola (Fabricius, 1781) & 2 & 0,32 & nd & $\mathrm{r}$ & $\mathrm{pf}$ & $\mathrm{z}$ \\
\hline Eurysthea hirta (Kirby, 1818) & 20 & 3,16 & d & ma & $\mathrm{mf}$ & $\mathrm{w}$ \\
\hline Eurysthea lacordairei (Lacordaire, 1869) & 1 & 0,16 & nd & $\mathrm{r}$ & $\mathrm{pf}$ & $\mathrm{z}$ \\
\hline Hippopsis sp. & 1 & 0,16 & nd & $\mathrm{r}$ & $\mathrm{pf}$ & $\mathrm{z}$ \\
\hline Hyperplatys argentinus (Berg, 1889) & 2 & 0,32 & nd & $\mathrm{r}$ & $\mathrm{pf}$ & $\mathrm{z}$ \\
\hline Leptostylus obscurellus Bates, 1863 & 1 & 0,16 & nd & $\mathrm{r}$ & $\mathrm{pf}$ & $\mathrm{z}$ \\
\hline Lypsimena nodipennis (Burmeister, 1965) & 4 & 0,63 & nd & d & $\mathrm{pf}$ & $\mathrm{z}$ \\
\hline Mallocera glauca Audinet-Serville, 1833 & 1 & 0,16 & nd & $\mathrm{r}$ & $\mathrm{pf}$ & $\mathrm{z}$ \\
\hline Martinsellus signatus (Gyllenhal, 1817) & 1 & 0,16 & nd & $\mathrm{r}$ & $\mathrm{pf}$ & $\mathrm{z}$ \\
\hline Megacyllene acuta (Germar, 1821) & 5 & 0,79 & nd & $\mathrm{c}$ & $\mathrm{f}$ & $\mathrm{y}$ \\
\hline Nealcidion bicristatum (Bates, 1863) & 2 & 0,32 & nd & $\mathrm{r}$ & $\mathrm{pf}$ & $\mathrm{z}$ \\
\hline Neoclytus centurio (Chevrolat, 1862) & 17 & 2,69 & d & a & $\mathrm{mf}$ & $\mathrm{y}$ \\
\hline Neoclytus curvatus (Germar, 1821) & 270 & 42,72 & sd & sa & sf & $\mathrm{w}$ \\
\hline Neoclytus pusillus (Laporte \& Gory, 1836) & 16 & 2,53 & d & $\mathrm{c}$ & $\mathrm{f}$ & $\mathrm{y}$ \\
\hline Neoclytus ypsilon Chevrolat, 1865 & 16 & 2,53 & d & $\mathrm{c}$ & $\mathrm{f}$ & $\mathrm{y}$ \\
\hline Neodillonia adspersa (Germar, 1824) & 1 & 0,16 & nd & $\mathrm{r}$ & $\mathrm{pf}$ & $\mathrm{z}$ \\
\hline Nyssodrysina lignaria (Bates, 1864) & 102 & 16,14 & d & ma & $\mathrm{mf}$ & $\mathrm{w}$ \\
\hline Oreodera aerumnosa Erichson, 1847 & 1 & 0,16 & nd & $\mathrm{r}$ & $\mathrm{pf}$ & $\mathrm{z}$ \\
\hline Oxymerus luteus luteus (Voet,1778) & 3 & 0,47 & nd & d & $\mathrm{pf}$ & $\mathrm{y}$ \\
\hline Paromoecerus barbicornis (Fabricius, 1792) & 11 & 1,74 & d & $\mathrm{c}$ & $\mathrm{f}$ & $\mathrm{y}$ \\
\hline Phoracantha recurva Newman, 1840 & 1 & 0,16 & nd & $\mathrm{r}$ & $\mathrm{pf}$ & $\mathrm{z}$ \\
\hline Retrachyderes thoraxicus (Olivier, 1790) & 13 & 2,06 & d & $\mathrm{c}$ & $\mathrm{f}$ & $\mathrm{w}$ \\
\hline Thelgetra latipennis Thomson, 1864 & 1 & 0,16 & nd & $\mathrm{r}$ & $\mathrm{pf}$ & $\mathrm{z}$ \\
\hline Unxia gracilior (Burmeister, 1865) & 2 & 0,32 & nd & $\mathrm{r}$ & $\mathrm{pf}$ & $\mathrm{z}$ \\
\hline Total & 632 & 100,0 & - & - & - & - \\
\hline
\end{tabular}

$\mathrm{D}=$ Dominância: $\mathrm{sd}=$ superdominante; $\mathrm{d}=$ dominante $\mathrm{e}$ nd = não dominante

$\mathrm{A}=$ Abundância: $\mathrm{sa}=$ superabundante; $\mathrm{ma}=$ muito abundante; $\mathrm{a}=$ abundante; $\mathrm{r}=\mathrm{rara} ; \mathrm{c}=$ comum e $\mathrm{d}=$ dispersa

$\mathrm{F}=$ Frequência: $\mathrm{sf}=$ superfrequente; $\mathrm{mf}=$ muito frequente; $\mathrm{f}=$ frequente e $\mathrm{pf}=$ pouco frequente

$\mathrm{C}=$ Constância: $\mathrm{w}=$ constante $\mathrm{y}=$ acessória $\mathrm{e} \mathrm{z}=$ acidental

${ }^{1}$ Dominância: Método de Laroca e Mielke 
Bernardi, O. et al.

Por serem categorizadas faunisticamente como espécies dominantes, e levando-se em conta a citação de Silveira Neto et al. (1976) os quais afirmam que os insetos dominantes têm capacidade de modificar um impacto recebido do ambiente em benefício próprio, a expansão da eucaliptocultura e o aumento da oferta de alimento no sul do Rio Grande do Sul, pode favorecer o aumento populacional das espécies do gênero Neoclytus, podendo explicar danos desses insetos antes considerados de pouca ou nenhuma importância à eucaliptocultura do Rio Grande do Sul.

Dentre as espécies predominantes, Nyssodrysina lignaria foi a segunda espécie que apresentou maior quantidade de indivíduos coletados (Tabela 2). Mesmo ocorrendo em grande quantidade no cultivo de eucalipto, essa essência florestal não é conhecida como hospedeira desse inseto (MARINONI et al., 2002). A coleta de grande quantidade de insetos dessa espécie no cultivo de eucalipto pode ter sido influenciada pelo álcool usado como atrativo, o qual poderia ter sido o responsável pela atração de grande quantidade de insetos de fragmentos de mata nativa dos arredores onde os insetos poderiam estar se desenvolvendo em frutos de árvores nativas como espécies de ingá (Inga spp.) e posteriormente serem atraídos pelas armadilhas para os talhões de eucalipto.

Dos insetos considerados predominantes, destaca-se a espécie Acanthoderes jaspidea pela capacidade de se desenvolver sobre eucalipto. Esta foi detectada por Berti Filho (1985) e Zanuncio et al. (1993) em troncos de Eucalyptus spp., e por Monné et al. (2002) em árvores caídas de Eucalyptus globulus.

Das diversas espécies de cerambicídeos coletados também se destaca Phoracantha recurva, a qual possui capacidade de desenvolver-se sobre várias espécies de eucalipto, conforme constatado por Monné et al. (2002), em Eucalyptus grandis e Eucalyptus globulus spp. globulus nas Províncias de Rivera, Paysandú e Lavalleja no Uruguai. Essa espécie foi introduzida no Brasil, e sua presença no Rio Grande do Sul, segundo Silva e Krüger (2004), foi detectada no ano de 1994. No entanto, sua maior importância como praga reside no fato de que as larvas do gênero Phoracantha são consideradas pragas quarentenárias para o Brasil e países da Europa, podendo ocasionar problemas à exportação de toras de eucalipto cuja constatação ou vestígios de danos podem resultar no embargo da madeira exportada (RIBEIRO et al., 2001).
Cerambicídeos como Chlorida festiva, Chydarteres striatus striatus, Paromoecerus barbicornis e Retrachyderes thoraxicus foram consideradas espécies dominantes, constantes e frequentes (Tabela 2). Destas, Retrachyderes thoraxicus foi mencionada broqueando Eucalyptus sp. por Berti Filho (1985), Eucalyptus citriodora, Eucalyptus tereticornis e Eucalyptus viminalis (MORAES e BERTI FILHO, 1974).

Os cerambicídeos Centrocerum elegans, Compsoceridius gounellei, Compsocerus violaceus, Dorcacerus barbatus, Erosida lineola, Eurysthea lacordairei, Hippopsis sp., Hyperplatys argentinus, Leptostylus obscurellus, Martinsellus signatus, Nealcidion bicristatum, Neodillonia adspersa, Oreodera aerumnosa, Phoracantha recurva, Thelgetra latipennis e Unxia gracilior foram consideradas espécies raras (Tabela 1).

Destas, Compsoceridius gounellei $e$ Thelgetra latipennis foram encontradas no Uruguai desenvolvendo-se em Eucalyptus grandis e Eucalyptus globulus conforme relato de Monné et al. (2002) e Unxia gracilior em Eucalyptus spp. (MONNÉ, 1970).

De acordo com Krebs (1972), as espécies raras exprimem à alta diversidade de um sistema que evolui em ambiente de sucessão ecológica, tendo as comunidades a característica marcante de conter grande número de espécies raras, no entanto, esse número pode diminuir quando se despende maior esforço amostral. Isso é reforçado por Espadaler e López-Soria (1991) que atribui a raridade a dois fatores: à dificuldade de coleta e à baixa ocorrência de insetos nos ambientes estudados.

Levando-se em consideração, a afirmação de Zanuncio et al. (1994), que o incremento da área reflorestada com essências exóticas tem levado ao aumento de problemas com insetospraga, em razão das modificações ambientais, nas monoculturas, que levam ao empobrecimento geral da fauna, há possibilidade de que alguma espécie de Cerambycidae venha a se estabelecer na cultura do eucalipto no sul do Rio Grande do Sul e ocasione danos em determinadas épocas, especialmente nos períodos do ano com temperatura elevada.

\section{CONCLUSÕES}

Os Cerambycidae coletados em florestamento de Eucalyptus spp., no município de Pinheiro Machado, estão distribuídos em 29 gêneros e 40 espécies.

Ci. Fl., v. 21, n. 1, jan.-mar., 2011 
As espécies mais abundantes de cerambicídeos em Eucalyptus spp. no município de Pinheiro Machado são Acanthoderes jaspidea, Chlorida costata, Compsocerus barbicornis, Eburodacrys sp., Eurysthea hirta, Neoclytus curvatus e Nyssodrysina lignaria.

O pico populacional de Cerambycidae no sul do Rio Grande do Sul ocorre no mês de dezembro.

Dos cerambicídeos coletados, a espécie Neoclytus curvatus necessita ser monitorada por causa da elevada abundância, frequência de coleta e potencial de danos às espécies do gênero Eucalyptus.

\section{REFERÊNCIAS BIBLIOGRÁFICAS}

ABRAF, 2008. Associação brasileira de produtores de florestas plantadas. Anuário estatístico 2008 (Ano base 2007). Disponível em: $\quad<$ (http://www.abraflor.org.br/estatística.asp) $>$ Acesso em: 28 de março de 2009.

ANJOS, N.; SANTOS, G. P.; ZANUNCIO, J. C. Pragas do eucalipto e seu controle. Informe Agropecuário, Belo Horizonte, v. 12, n. 141, p. 5058, set./out. 1986.

BERTI FILHO, E. Insects associated to eucalipt plantations in Brazil. In: IUFRO WP. S2.07.07. PROTECTION OF FOREST IN THE TROPICS. Noxious insects to Pine and Eucalypt plantations in the tropics. Curitiba: Universidade Federal do Paraná, 1985, p. 162-178.

BERTI FILHO, E. Impacto de Coleoptera Cerambycidae em florestas de Eucalyptus no Brasil. Scientia Forestalis, Piracicaba, v. 52, p. 51-54, dez. 1997.

BIEZANKO, C. M.; BOSQ, J. M. Cerambycidae de Pelotas e seus arredores. Agros, Pelotas, v. 9, n. 3/4, p. 3-15, mar. 1956.

CARVALHO, A. O. R. Análise faunística de coleópteros coletados em plantas de Eucalyptus urophylla S.T. Blake e Eucalyptus saligna Sm. 1984. 105 f. Dissertação (Mestrado em Ciências/ Entomologia)-Universidade de São Paulo. Escola Superior de Agricultura "Luiz de Queiroz", Piracicaba, 1984.

COSTA, E. C. D; LINK, D. Flutuação populacional de Nyssodrysina lignaria (Bates, 1864) (Coleoptera, Cerambycidae). In: CONGRESSO FLORESTAL ESTADUAL, 1988. Anais... Nova Prata: Prefeitura Municipal, 1988, p. 541-550.

ESPADALER, X.; LÓPEZ-SORIA, L. Rareness of certain mediterranean ant species: factor or artifact?
Insectes Sociaux, Leuven, v. 38, n. 4, p. 365-377, Dec. 1991.

FLECHTMANN, C. A. H. et al. Manual de pragas em florestas - Scolytidae em reflorestamento com pinheiros tropicais. Piracicaba, Brasil: Programa Cooperativo de Manejo de Pragas Florestais - IPEF, 1995. $201 \mathrm{p}$.

FLECHTMANN, C. A. H.; OTTATI, A. L. T.; BERISFORD, C. W. Comparison of four trap types for ambrosia beetles (Coleoptera: Scolytidae). Journal of Economic Entomology, Lanham, v. 93, n. 6, p. 1701-1707, Dec. 2000.

FREITAS, F. A. et al. Fauna de Coleoptera coletada com armadilhas luminosas em um plantio de Eucalyptus grandis em Santa Bárbara, Minas Gerais. Revista Árvore, Viçosa, v. 26, n. 4, p. 505511, jul./ago. 2002.

GARCIA, F. R. M.; CORSEUIL, E. Flutuação populacional de cerambicídeos e escarabeídeos (Coleoptera) em pomares de pessegueiro no município de Porto Alegre, Rio Grande do Sul. Revista da Faculdade de Zootecnia, Veterinária e Agronomia, Uruguaiana, v. 5/6, n. 1, p. 69-81, mar. 1998.

KREBS, C. J. Ecology: The experimental analysis of distribution and abundance, 2nd ed. Cambridge: Harper and Row, 1972, 694 p.

MARINONI, R. C.; GANHO, N. G.; RIBEIROCOSTA, C. S. Feeding habits of Nyssodrysina lignaria (Bates) (Coleoptera: Cerambycidae: Lamiinae). Proceedings of the Entomological Society of Washington, Washington, v. 104, n. 4, p. 817-819, Oct. 2002.

MARINONI, R. C.; GANHO, N. G. Sazonalidade de Nyssodrysina lignaria (Bates) (Coleoptera, Cerambycidae, Lamiinae) no Estado do Paraná, Brasil. Revista Brasileira de Zoologia, Curitiba, $n$. 20, v. 1, p. 141-152, mar. 2003.

MARTINS, U. R. Cerambycidae Sul-Americanos (Coleoptera) - III. Subfamíia Cerambycinae, Hesperophanini Mulsant, 1839; Eburiini Blanchard, 1845; Diorini Lane, 1950. São Paulo, $1999,418 \mathrm{p}$.

MARTINS, U. R. Cerambycidae Sul-Americanos (Coleoptera) - VII. Subfamília Cerambycinae, Elaphidionini Thamson 1864. São Paulo, 2005. $394 \mathrm{p}$.

MONNÉ, M. Fauna de los coleópteros del Uruguay. 1970, 216 p. Tesis (Doctor en Ciencias) - Universidade de la República. Faculdad de Agronomía. Montivideo.

MONNÉ, M. et al. Cerambicídos (Coleoptera) que 
Bernardi, O. et al.

atacan Eucalyptus globulus y Eucalyptus grandis en Uruguay. Agrociencia, Coyoacán, v. 6, n. 1, p. 6368, jan./fev. 2002.

MORAES, G. J.; BERTI FILHO, E. Coleobrocas que ocorrem em essências florestais. IPEF, n. 9, p. 27-42, jan./fev. 1974.

MORAES, R. C. B. et al. Software para análise faunística - AnaFau. In: SIMPÓSIO DE CONTROLE BIOLÓGICO, 2003, São Pedro. Resumos... São Pedro, 2003. p. 195.

NETO, L. W.; LINK, D. Cerambycidae associados à Lauraceae, na região central do Rio Grande do Sul, Brasil. Ciência Florestal, Santa Maria, v. 7, n. 1, p. 33-39, jan./mar. 1997.

RIBEIRO, G. T. et al. Ocorrência da broca do eucalipto Phoracantha semipunctata Fabricius (Coleoptera: Cerambycidae), nos Estados de Minas Gerais, Paraná e Espírito Santo. Revista Árvore, Viçosa, v. 25, n. 3, p. 393-396, maio/jun. 2001.

SILVA, E. J. E.; KRÜGER, C. P. Phoracantha recurva (Newman, 1840) (Coleoptera: Cerambycidae) no Sul do Brasil. Entomologia y Vectores, Rio de Janeiro, v. 1, n. 1, p. 69-76, jan./ mar. 2004.

SILVEIRA NETO, S. et al. Manual de ecologia dos insetos. Piracicaba: Ceres, $1976.419 \mathrm{p}$.

WOLDAN, D. R. H. Análise faunística de Cerambycidae (Coleoptera) em duas situações florísticas no município de União da Vitória, Paraná. 2007. 64 f. Dissertação (Mestrado em Ciências Ambientais)-Universidade Comunitária Regional de Chapecó, Chapecó, 2007.

ZANUNCIO, J. C. et al. Coleópteros associados à eucaliptocultura nas regiões de São Mateus e Aracruz, Espírito Santo. Revista Ceres, Viçosa, v. 41, n. 22, p. 584-90, nov./dez. 1993.

ZANUNCIO, J. C. et al. Major lepidopterous defoliators of eucalypt in southeast Brazil. Forest Ecology and Management, Amsterdam, v. 65, p. 53-63, May 1994. 